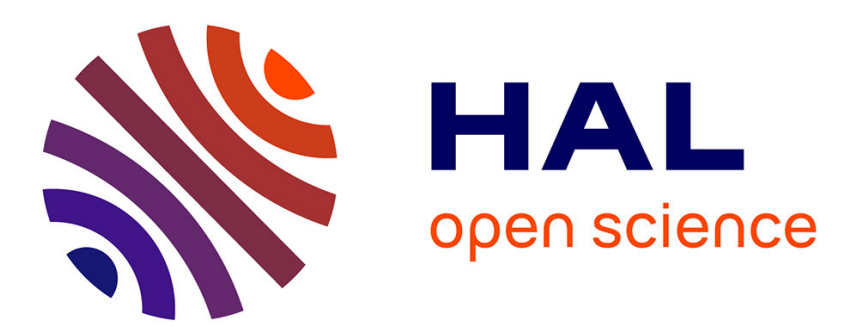

\title{
Multi-valued boundary value problems involving Leray-Lions operators and discontinuous nonlinearities
}

Simona Dabuleanu, Vicentiu Radulescu

\section{To cite this version:}

Simona Dabuleanu, Vicentiu Radulescu. Multi-valued boundary value problems involving Leray-Lions operators and discontinuous nonlinearities. 2003. hal-00000188

\section{HAL Id: hal-00000188 \\ https://hal.science/hal-00000188}

Preprint submitted on 13 Feb 2003

HAL is a multi-disciplinary open access archive for the deposit and dissemination of scientific research documents, whether they are published or not. The documents may come from teaching and research institutions in France or abroad, or from public or private research centers.
L'archive ouverte pluridisciplinaire HAL, est destinée au dépôt et à la diffusion de documents scientifiques de niveau recherche, publiés ou non, émanant des établissements d'enseignement et de recherche français ou étrangers, des laboratoires publics ou privés. 


\title{
MULTI-VALUED BOUNDARY VALUE PROBLEMS INVOLVING LERAY-LIONS OPERATORS AND DISCONTINUOUS NONLINEARITIES
}

\author{
SIMONA DĂBULEANU - VICENŢIU RĂDULESCU
}

\begin{abstract}
We prove an existence result for a class of Dirichlet boundary value problems with discontinuous nonlinearity and involving a Leray-Lions operator. The proof combines monotonicity methods for elliptic problems, variational inequality techniques and basic tools related to monotone operators. Our work generalizes a result obtained in Carl [4].

Key words: sub- and super-solution, Leray-Lions operator, maximal monotone graph, pseudo-monotone operator, variational inequality.
\end{abstract}

2000 Mathematics Subject Classification: 35J65, 47H10, 47J25, 58 J32.

\section{Introduction and the main result.}

Let $\Omega \subset \mathbf{R}^{N}$ be a bounded domain with smooth boundary. Consider the boundary value problem

$$
\text { (P) } \begin{cases}-\operatorname{div}(a(x, \nabla u(x)))=f(u(x)), & \text { if } x \in \Omega \\ u=0, & \text { on } \partial \Omega,\end{cases}
$$

where $a: \Omega \times \mathbf{R}^{N} \rightarrow \mathbf{R}^{N}$ is a Carathéodory function having the properties

$\left(a_{1}\right)$ there exist $p>1$ and $\lambda>0$ such that $a(x, \xi) \cdot \xi \geq \lambda \cdot\|\xi\|^{p}$, for a.e. $x \in \Omega$ and for any $\xi \in \mathbf{R}^{N}$;

$\left(a_{2}\right)(a(x, \xi)-a(x, \eta)) \cdot(\xi-\eta)>0$, for any $\xi, \eta \in \mathbf{R}^{N}, \xi \neq \eta$;

$\left(a_{3}\right)$ there exist $\alpha \in \mathbf{R}^{+}$and $k \in L^{p^{\prime}}(\Omega)$ such that $|a(x, \xi)| \leq \alpha\left(k(x)+|\xi|^{p-1}\right)$, for a.e. $x \in \Omega$ and for any $\xi \in \mathbf{R}^{N}$.

Assume that the nonlinearity $f: \mathbf{R} \rightarrow \mathbf{R}$ satisfies the hypothesis

$\left(H_{1}\right)$ there exist nondecreasing functions $f, g: \mathbf{R} \rightarrow \mathbf{R}$ such that $f=g-h$. 
Let $\beta: \mathbf{R} \rightarrow 2^{\mathbf{R}}$ be the maximal monotone graph associated with the nondecreasing function $h$ (see Brezis [3]). More exactly,

$$
\beta(s):=\left[h^{-}(s), h^{+}(s)\right], \quad \text { for all } s \in \mathbf{R},
$$

where

$$
h^{-}(s)=\lim _{\varepsilon \rightarrow 0+} h(s-\varepsilon), \quad h^{+}(s)=\lim _{\varepsilon \rightarrow 0+} h(s+\varepsilon) .
$$

Under this assumption we reformulate the problem $(P)$ as follows

$$
\left(P^{\prime}\right) \begin{cases}-\operatorname{div}(a(x, \nabla(x)))+\beta(u(x)) \ni g(u(x)), & \text { if } x \in \Omega \\ u=0, & \text { on } \partial \Omega .\end{cases}
$$

Denote by $G$ the Nemitskii operator associated with $g$, that is, $G(u)(x)=$ $g(u(x))$.

DEFINITION 1. A function $u \in W_{0}^{1, p}(\Omega)$ is called a solution of the problem $\left(P^{\prime}\right)$ if there exists $v \in L^{p^{\prime}}(\Omega)$ such that

i) $v(x) \in \beta(u(x))$ a.e. in $\Omega$,

ii) $\int_{\Omega} a(x, \nabla u) \cdot \nabla w d x+\int_{\Omega} v \cdot w d x=\int_{\Omega} G(u) \cdot w d x$, for any $w \in W_{0}^{1, p}(\Omega)$.

Let $L_{+}^{p}$ be the set of nonnegative elements of $L^{p}(\Omega)$. For any $v, w \in \Omega$ such that $v \leq w$, we set

$$
[v, w]=\left\{u \in L^{p}(\Omega) / v \leq u \leq w\right\} .
$$

DEFINITION 2. A function $\bar{u} \in W^{1, p}(\Omega)$ is called an upper solution of the problem $\left(P^{\prime}\right)$ if there exists a function $\bar{v} \in L^{p^{\prime}}(\Omega)$ such that

i) $\bar{v}(x) \in \beta(\bar{u}(x))$ a.e. in $\Omega$,

ii) $\bar{u} \geq 0$ on $\partial \Omega$,

iii) $\int_{\Omega} a(x, \nabla \bar{u}) \cdot \nabla w d x+\int_{\Omega} \bar{v} \cdot w d x \geq \int_{\Omega} G(\bar{u}) \cdot w d x$ for all

$w \in W_{0}^{1, p}(\Omega) \cap L_{+}^{p}(\Omega)$.

DEFINITION 3. A function $\bar{u} \in W^{1, p}(\Omega)$ is called a lower solution of the problem $\left(P^{\prime}\right)$ if there exists a function $\bar{v} \in L^{p^{\prime}}(\Omega)$ such that

i) $\underline{v}(x) \in \beta(\underline{u}(x))$ a.e. in $\Omega$, 
ii) $\underline{u} \leq 0$ on $\partial \Omega$,

iii) $\int_{\Omega} a(x, \nabla \underline{u}) \cdot \nabla w d x+\int_{\Omega} \underline{v} \cdot w d x \leq \int_{\Omega} G(\underline{u}) \cdot w d x$ for any $w \in W_{0}^{1, p}(\Omega) \cap L_{+}^{p}(\Omega)$.

In the sequel the following hypothesis will be needed:

$\left(H_{2}\right)$ There exist an upper solution $\bar{u}$ and a lower solution $\underline{u}$ of the problem $\left(P^{\prime}\right)$ such that $\underline{u} \leq \bar{u}$, and $G(\underline{u}), G(\bar{u}), H^{+}(\bar{u}), H^{-}(\underline{u}) \in L^{p^{\prime}}(\Omega)$.

The following is a generalization of the main result in Carl [4].

THEOREM 1. Assume hypothesis $\left(\mathrm{H}_{1}\right)$ and $\left(\mathrm{H}_{2}\right)$ hold and that $\mathrm{g}$ is right (resp. left) continuous. Then there exists a maximal (resp. minimal) solution $u \in[\underline{u}, \bar{u}]$ of the problem $\left(P^{\prime}\right)$.

\section{Proof of Theorem 1.}

We first reformulate the problem ( $\left.\mathrm{P}^{\prime}\right)$ in terms of variational inequalities using the subdifferential theory in the sense of convex analysis.

Let $j: \mathbf{R} \rightarrow(-\infty, \infty]$ be a convex, proper and lower semicontinuous function. Let $\partial j$ be the subdifferential of $j$, that is

$$
\partial j(r)=\{\hat{r} \in \mathbf{R}: j(s) \geq j(r)+\hat{r}(s-r) \quad \text { for all } s \in \mathbf{R}\} .
$$

We recall the following result concerning maximal monotone graphs in $\mathbf{R}^{2}$ (see Brezis [3] [Corollary 2.10], p. 43)

LEMMA 1. Let $\beta: \mathbf{R} \rightarrow 2^{\mathbf{R}}$ be a maximal monotone graph in $\mathbf{R}^{2}$. Then there exists a convex, proper and lower semicontinuous function $j: \mathbf{R} \rightarrow$ $(-\infty,+\infty]$ such that $\beta=\partial j$. Moreover, the function $j$ is uniquely determined up to an additive constant.

We observe that the function $h$ appearing in $\left(H_{1}\right)$ can always be chosen so that $h(0)=0$. Then the maximal monotone graph $\beta$ has the properties

$$
D(\beta)=\mathbf{R} \text { and } \quad 0 \in \beta(0) .
$$

Since the function $j$ related to $\beta$ according to Lemma 1 is uniquely determined up to an additive constant we can assume that

$$
j(0)=0 .
$$

So, by (1), (2) and (3) it follows that 


$$
j(s) \geq 0 \quad \text { for all } s \in \mathbf{R} .
$$

Define $J: L^{p}(\Omega) \rightarrow(-\infty,+\infty]$ by

$$
J(v)= \begin{cases}\int_{\Omega} j(v(x)) d x, & \text { for } j(v(\cdot)) \in L^{1}(\Omega) \\ +\infty & \text { otherwise. }\end{cases}
$$

Then $J$ is convex, proper and lower semicontinuous (see Barbu [1]).

Under the above assertions we can reformulate the problem $\left(P^{\prime}\right)$ in terms of variational inequalities as follows: find $u \in W_{0}^{1, p}(\Omega)$ such that

$$
\begin{aligned}
& \int_{\Omega} a(x, \nabla u) \cdot \nabla(w-u) d x+J(w)-J(u) \geq \int_{\Omega} G(u)(w-u) d x \\
& \text { for all } w \in W_{0}^{1, p}(\Omega) \text {. }
\end{aligned}
$$

LEMMA 2. Let hypotheses $\left(H_{1}\right)$ and $\left(H_{2}\right)$ be fulfilled. Then $u \in[\underline{u}, \bar{u}]$ is a solution of (5) if and only if $u$ is a solution of the problem $\left(P^{\prime}\right)$.

Proof. Let $u \in[\underline{u}, \bar{u}]$ satisfy the variational inequality (5). Then

$$
J(w) \geq J(u)+\int_{\Omega} G(u) \cdot(w-u) d x-\int_{\Omega} a(x, \nabla u) \cdot \nabla(w-u) d x .
$$

It follows that

$$
\operatorname{div}(a(x, \nabla u))+G(u) \in \partial J(u) \quad \text { in } W^{-1, p^{\prime}}(\Omega) .
$$

It follows by Brezis [2] [Corollaire 1] that any subgradient $v \in \partial J(u)$ of the functional $J: W_{0}^{1, p}(\Omega) \rightarrow(-\infty,+\infty]$ at $u \in W_{0}^{1, p}(\Omega)$ belongs to $L^{1}(\Omega)$ and satisfies

$$
v(x) \in \partial j(u(x))=\beta(u(x)) \quad \text { a.e. in } \Omega .
$$

Furthermore

$$
h^{-}(\underline{u}(x)) \leq h^{-}(u(x)) \leq \beta(u(x)) \leq h^{+}(\bar{u}(x)) \leq h^{+}(\bar{u}(x)) \quad \text { a.e. in } \Omega .
$$

Thus

$$
|v| \leq\left|H^{+}(\bar{u})\right|+\left|H^{-}(\underline{u})\right| .
$$

By $\left(H_{2}\right)$, the right-hand side of (8) belongs to $L^{p^{\prime}}(\Omega)$. It follows that $v \in$ $L^{p^{\prime}}(\Omega)$. Thus there exists $v \in L^{p^{\prime}}(\Omega)$ such that

$$
\operatorname{div}(a(x, \nabla u))+G(u)=v \quad \text { in } W^{-1, p^{\prime}}(\Omega)
$$


or, equivalently,

$$
\begin{aligned}
& \int_{\Omega} a(x, \nabla u) \cdot \nabla w d x+\int_{\Omega} v \cdot w d x=\int_{\Omega} G(u) w d x \\
& \text { for all } w \in W_{0}^{1, p}(\Omega) .
\end{aligned}
$$
$\left(P^{\prime}\right)$.

Relations (7) and (9) imply that $u \in W_{0}^{1, p}(\Omega)$ is a solution of the problem

Conversely, let $u \in[\underline{u}, \bar{u}]$ be a solution of the problem $\left(P^{\prime}\right)$. Then there exists $v \in L^{p^{\prime}}(\Omega)$ such that $v \in \beta(u)=\partial j(u(x))$ and the relation (9) is fulfilled. Since $v(x) \in \partial j(u(x))$ we have

$$
j(s) \geq j(u(x))+v(x)(s-u(x)) .
$$

Taking $s=0$ in (10) we obtain, by means of (3) and (4) that $0 \leq j(u(x)) \leq$ $v(x) u(x)$. Thus

$$
j(u(\cdot)) \in L^{1}(\Omega) \quad \text { and } \quad J(u)=\int_{\Omega} j(u(x)) d x .
$$

Let $w \in W_{0}^{1, p}(\Omega)$. Taking $s=w(x)$ in (10) we obtain

$$
\int_{\Omega} j(w(x)) d x-\int_{\Omega} j(u(x)) d x \geq \int_{\Omega} v(x)(w(x)-u(x)) d x .
$$

From (9), substituting $w$ by $w-u \in W_{0}^{1, p}(\Omega)$ we get, by means of (12)

$$
\begin{aligned}
\int_{\Omega} a(x, \nabla u) \cdot \nabla(w-u) d x+J(w)-J(u) \geq \int_{\Omega} G(u) \cdot(w-u) d x \\
\quad \text { for all } w \in W_{0}^{1, p}(\Omega) .
\end{aligned}
$$

This means that $u$ is a solution of the variational inequality (5).

Remark 1. If $u$ is a solution of $\left(P^{\prime}\right)$ then, by (11), $J(u)<+\infty$. The result also holds also if we replace $u$ by a super-solution $\bar{u}$ or by a sub-solution $\underline{u}$.

Set $v^{+}=\max \{v, 0\}$.

LEMMA 3. Let $u, v \in L^{p}(\Omega)$ such that $J(u)$ and $J(v)$ are finite. Then

$$
J\left(u-(u-v)^{+}\right)-J(u)+J\left(v+(u-v)^{+}\right)-J(v)=0 .
$$


Proof. Let $\Omega_{+}:=\{x \in \Omega \mid u>v\}$ and $\Omega_{-}:=\{x \in \Omega \mid u \leq v\}$. Since $(u-v)^{+}=0$ in $\Omega_{-}$and $(u-v)^{+}=u-v$ in $\Omega_{+}$we obtain

$$
\begin{aligned}
& J\left(u-(u-v)^{+}\right)=\int_{\Omega_{+}} j(v) d x+\int_{\Omega_{-}} j(u) d x \leq \infty \\
& J\left(v+(u-v)^{+}\right)=\int_{\Omega_{+}} j(u) d x+\int_{\Omega_{-}} j(v) d x \leq \infty
\end{aligned}
$$

By (14) and (15) we obtain (13).

Consider now the following variational inequality: given $z \in L^{p}(\Omega)$, find $u \in W_{0}^{1, p}(\Omega)$ such that

$$
\begin{aligned}
\int_{\Omega} a(x, \nabla u) \cdot \nabla(w-u)+J(w)-J(u) \geq \int_{\Omega} G(z)(w-u) d x \\
\quad \text { for all } w \in W_{0}^{1, p}(\Omega) .
\end{aligned}
$$

The variational inequality (16) defines a mapping $T: z \rightarrow u$ and each fixed point of $T$ yields a solution of (5) and conversely.

LEMMA 4. Let hypotheses $\left(H_{1}\right)$ and $\left(H_{2}\right)$ be satisfied. Then for each $z \in$ $[\underline{u}, \bar{u}]$ the variational inequality (16) has a unique solution $u=T z \in[\underline{u}, \bar{u}]$. Moreover, there is a constant $C>0$ such that $\|T z\|_{W_{0}^{1, p}(\Omega)} \leq C$, for any $z \in[\underline{u}, \bar{u}]$.

Proof. Existence. Let $z \in[\underline{u}, \bar{u}]$ be arbitrarily given. Then $G(z)$ is measurable and $G(z) \in L^{p^{\prime}}(\Omega)$, due to the estimate

$$
|G(z)| \leq|G(\bar{u})|+|G(\underline{u})|
$$

and after observing that the right-hand side of the above inequality is in $L^{p^{\prime}}(\Omega)$, by $\left(H_{2}\right)$.

We now apply Theorem II.8.5 in Lions [5]. We first observe that the above assertions show that the mapping $W_{0}^{1, p}(\Omega) \ni u \rightarrow \int_{\Omega} G(z) u$ is in $W^{-1, p^{\prime}}(\Omega)$.

Consider the Leray-Lions operator $A: W_{0}^{1, p}(\Omega) \rightarrow W^{-1, p^{\prime}}(\Omega)$ defined by

$$
\langle A u, w\rangle=\int_{\Omega} a(x, \nabla u) \cdot \nabla w d x .
$$


We show that $A$ is a pseudo-monotone operator. For this aim it is enough to prove that $A$ is bounded, monotone and hemi-continuous (see Lions [5] [Prop. II.2.5]).

Condition $\left(a_{3}\right)$ yields the boundedness of $A$. Indeed

$$
\|A u\|_{W^{-1, p^{\prime}}}(\Omega) \leq C\left(\|k\|_{L^{p^{\prime}(\Omega)}}+\|\nabla u\|_{L^{p}(\Omega)}^{p-1}\right) .
$$

We also observe that $\left(a_{2}\right)$ implies that $A$ is a monotone operator.

In order to justify the hemi-continuity of $A$, let us consider a sequence $\left(\lambda_{n}\right)_{n \geq 1}$ converging to $\lambda$. Then, for given $u, v, w \in W_{0}^{1, p}(\Omega)$, we have

$$
a\left(x, \nabla\left(u+\lambda_{n} v\right)\right) \cdot \nabla w \rightarrow a(x, \nabla(u+\lambda v)) \cdot \nabla w \quad \text { a.e. in } \Omega .
$$

From the boundedness of $\left\{\lambda_{n}\right\}$ and condition $\left(a_{3}\right)$ we obtain that the sequence $\left\{\left|a\left(x, \nabla\left(u+\lambda_{n} v\right)\right) \nabla w\right|\right\}$ is bounded by a function which belongs to $L^{1}(\Omega)$. Using the Lebesgue dominated convergence theorem it follows that

$$
\left\langle A\left(u+\lambda_{n} v\right), w\right\rangle \rightarrow\left\langle A\left(u+\lambda_{n} v\right), w\right\rangle \quad \text { as } n \rightarrow \infty .
$$

Hence the application $\lambda \rightarrow\langle A(u+\lambda v, w\rangle)$ is continuous.

It follows that all assumptions of Theorem II.8.5 in [5] are fulfilled, so the problem (16) has at least a solution.

Uniqueness. Let $u_{1}$ and $u_{2}$ be two solutions of (16). Then taking $w=u_{2}$ as a test function for the solution $u_{1}$, we obtain

$$
\int_{\Omega} a\left(x, \nabla u_{1}\right) \cdot \nabla\left(u_{2}-u_{1}\right) d x+J\left(u_{2}\right)-J\left(u_{1}\right) \geq \int_{\Omega} G(z)\left(u_{2}-u_{1}\right) d x .
$$

Similarly we find

$$
\int_{\Omega} a\left(x, \nabla u_{2}\right) \cdot \nabla\left(u_{1}-u_{2}\right) d x+J\left(u_{1}\right)-J\left(u_{2}\right) \geq \int_{\Omega} G(z)\left(u_{1}-u_{2}\right) d x .
$$

Therefore

$$
\int_{\Omega}\left(a\left(x, \nabla u_{1}\right)-a\left(x, \nabla u_{2}\right)\right) \cdot\left(\nabla u_{1}-\nabla u_{2}\right) d x \leq 0 .
$$

So, by $\left(a_{2}\right)$, it follows that $\nabla u_{1}=\nabla u_{2}$, so $u_{1}=u_{2}+C$ in $\Omega$. Since $u_{1}=u_{2}=0$ on $\partial \Omega$, it follows that $u_{1}=u_{2}$ in $\Omega$.

From (3) and (4) we deduce that $J(0)=0$ and $J(u) \geq 0$. Moreover, the variational inequality (16) implies

$$
\int_{\Omega} a(x, \nabla u) \cdot \nabla(-u) d x+J(0)-J(u) \geq-\int_{\Omega} G(z) u d x .
$$


Thus

$$
\int_{\Omega} a(x, \nabla u) \cdot \nabla u d x \leq \int_{\Omega} G(z) u d x .
$$

This last inequality, assumption $\left(a_{1}\right)$ and Hölder's inequality yield

$$
\begin{aligned}
\lambda \cdot\|u\|_{W_{0}^{1, p}(\Omega)}^{p} & \leq \int_{\Omega} G(z) u d x \leq\|G(z)\|_{L^{p^{\prime}}(\Omega)} \cdot\|u\|_{L^{p}} \\
& \leq C_{1}\left(\|G(\bar{u})\|_{L^{p^{\prime}}(\Omega)}+\|G(\underline{u})\|_{L^{p^{\prime}}(\Omega)}\right)\|u\|_{W_{0}^{1, p}(\Omega)} .
\end{aligned}
$$

Thus $u=T z$ verifies

$$
\|u\|_{W_{0}^{1, p}(\Omega)}^{p-1} \leq C_{1}\left(\|G(\bar{u})\|_{L^{p^{\prime}}(\Omega)}+\|G(\underline{u})\|_{L^{p^{\prime}}(\Omega)}\right)=C_{2} .
$$

This implies that there exists a universal constant $C>0$ such that

$$
\|u\|_{W_{0}^{1, p}(\Omega)} \leq C .
$$

So, in order to conclude our proof, it is enough to show that $u \in[u, \bar{u}$. But, by the definition of an upper solution, there exists $\bar{v} \in L^{p^{\prime}}(\Omega)$ such that $\bar{v} \in \beta(\bar{u}(x))$ and

$$
\begin{aligned}
\int_{\Omega} a(x, \nabla \bar{u}) \cdot \nabla w d x+\int_{\Omega} \bar{v} \cdot w d x \geq \int_{\Omega} G(\bar{u}) w d x, \\
\text { for all } w \in W_{0}^{1, p}(\Omega) \cap L_{+}^{p}(\Omega) .
\end{aligned}
$$

The solution $u=T z$ of the variational inequality (16) satisfies

$$
\begin{aligned}
& \int_{\Omega} a(x, \nabla u) \cdot \nabla(w-u) d x+J(w)-J(u) \geq \int_{\Omega} G(z)(w-u) d x \\
& \text { for all } w \in W_{0}^{1, p}(\Omega) \text {. }
\end{aligned}
$$

Setting $\bar{v} \in \beta(\bar{u})=\partial j(\bar{u})$, we have

$$
j(s) \geq j(\bar{u}(x))+\bar{v}(x)(s-\bar{u}(x)) \quad \text { for all } s \in \mathbf{R} .
$$

Taking $s:=\bar{u}(x)+(u(x)-\bar{u}(x))^{+}$in (19) we find by integration

$$
J\left(\bar{u}+(u-\bar{u})^{+}\right) \geq J(\bar{u})+\int_{\Omega} \bar{v}(u-\bar{u})^{+} d x .
$$


Choosing now $w=(u-\bar{u})^{+}$in (17) we obtain

(21) $\int_{\Omega} a(x, \nabla \bar{u}) \cdot \nabla(u-\bar{u})^{+} d x+\int_{\Omega} \bar{v} \cdot(u-\bar{u})^{+} d x \geq \int_{\Omega} G(\bar{u}) \cdot(u-\bar{u})^{+} d x$.

Relations (20) and (21) yield

(22) $\int_{\Omega} a(x, \nabla \bar{u}) \cdot \nabla(u-\bar{u})^{+} d x+J\left(\bar{u}+(u-\bar{u})^{+}\right)-J(\bar{u}) \geq \int_{\Omega} G(\bar{u}) \cdot(u-\bar{u})^{+} d x$.

Taking $w=u-(u-\bar{u})^{+}$in (18), we obtain

$\int_{\Omega} a(x, \nabla u) \cdot\left(-\nabla(u-\bar{u})^{+}\right) d x+J\left(u-(u-\bar{u})^{+}\right)-J(u) \geq-\int_{\Omega} G(z)(u-\bar{u})^{+} d x$.

Since $z \in[\underline{u}, \bar{u}]$ and $G: L^{p}(\Omega) \rightarrow L^{p}(\Omega)$ is nondecreasing, it follows that

$$
\begin{aligned}
\int_{\Omega} a(x, \nabla u) \cdot \nabla(u-\bar{u})^{+} d x & +J\left(u-(u-\bar{u})^{+}\right)-J(u) \\
\geq & -\int_{\Omega} G(\bar{u})(u-\bar{u})^{+} d x .
\end{aligned}
$$

From (22), (23) and Lemma 3 we have

$$
\int_{\Omega}\left(a(x, \nabla u)-a(x, \nabla \bar{u}) \cdot \nabla(u-\bar{u})^{+} d x \leq 0 .\right.
$$

Let $\Omega_{+}=\{x \in \Omega \mid u \leq \bar{u}\}$ and $\Omega_{-}=\{x \in \Omega \mid u>\bar{u}\}$. Since $(u-\bar{u})^{+}=0$ in $\Omega_{+}$and $(u-\bar{u})^{+}=u-\bar{u}$ in $\Omega_{-}$, it follows by (24) that

$$
\int_{\Omega_{-}}\left(a(x, \nabla u)-a(x, \nabla \bar{u}) \cdot \nabla(u-\bar{u})^{+} d x \leq 0 .\right.
$$

So, by $\left(a_{2}\right)$ and the definition of $\Omega_{-}$, we obtain meas $\left(\Omega^{-}\right)=0$, hence $u \leq \bar{u}$ a.e. in $\Omega$. Proceeding in the same way we prove that $\underline{u} \leq u$.

LEMMA 5. The operator $T$ defines a monotone nondecreasing mapping from $[\underline{u}, \bar{u}]$ to $[\underline{u}, \bar{u}]$. 
Proof. Let $z_{1}, z_{2} \in[\underline{u}, \bar{u}]$ be such that $z_{1} \leq z_{2}$. By Lemma 4, we obtain that $T z_{1}, T z_{2} \in[\underline{u}, \bar{u}]$ and

$$
\begin{aligned}
\int_{\Omega} a\left(x, \nabla T z_{1}\right) \cdot \nabla\left(w-T z_{1}\right) d x & +J(w)-J\left(T z_{1}\right) \\
& \geq \int_{\Omega} G\left(z_{1}\right)\left(w-T z_{1}\right) d x \\
\int_{\Omega} a\left(x, \nabla T z_{2}\right) \cdot \nabla\left(w-T z_{2}\right) d x & +J(w)-J\left(T z_{1}\right) \\
& \geq \int_{\Omega} G\left(z_{2}\right)\left(w-T z_{2}\right) d x .
\end{aligned}
$$

Taking $w=T z_{1}-\left(T z_{1}-T z_{2}\right)^{+}$in (25) and $w=T z_{2}+\left(T z_{1}-T z_{2}\right)^{+}$in (26), we get

$$
\begin{aligned}
-\int_{\Omega} a\left(x, \nabla T z_{1}\right) \cdot \nabla\left(T z_{1}-T z_{2}\right)^{+} d x & +J\left(T z_{1}-\left(T z_{1}-T z_{2}\right)^{+}\right)-J\left(T z_{1}\right) \\
& \geq \int_{\Omega} G\left(z_{1}\right)\left(-\left(T z_{1}-T z_{2}\right)^{+}\right) d x \\
\int_{\Omega} a\left(x, \nabla T z_{2}\right) \cdot \nabla\left(T z_{1}-T z_{2}\right)^{+} d x & +J\left(T z_{2}+\left(T z_{1}-T z_{2}\right)^{+}\right)-J\left(T z_{2}\right) \\
\geq & \int_{\Omega} G\left(z_{2}\right)\left(T z_{1}-T z_{2}\right)^{+} d x .
\end{aligned}
$$

Summing up these inequalities we get, by means of (13),

$$
\begin{aligned}
\int_{\Omega}\left(a\left(x, \nabla T z_{1}\right)\right. & \left.-a\left(x, \nabla T z_{2}\right)\right) \cdot \nabla\left(T z_{1}-T z_{2}\right)^{+} d x \\
& \leq \int_{\Omega}\left(G\left(z_{1}\right)-G\left(z_{2}\right)\right)\left(T z_{1}-T z_{2}\right)^{+} d x .
\end{aligned}
$$

But $G\left(z_{1}\right) \leq G\left(z_{2}\right)$, since $G$ is a nondecreasing operator. Therefore, by the 
above inequality we obtain

$$
\int_{\Omega} a\left(x,\left(\nabla T z_{1}\right)-a\left(x, \nabla T z_{2}\right)\right) \cdot \nabla\left(T z_{1}-T z_{2}\right)^{+} d x \leq 0 .
$$

With the same argument as for proving (24) we obtain $T z_{1} \leq T z_{2}$.

Proof of Theorem 1 completed. Assume that $g$ is right continuous. Define

$$
u^{n+1}=T u^{n},
$$

where $u^{0}=\bar{u}$. Then, by Lemma $4,\left\{u^{n}\right\}$ is nondecreasing, $u^{n} \in[\underline{u}, \bar{u}]$, and there is a constant $C$ such that

$$
\left\|u^{n}\right\|_{W_{0}^{1, p}(\Omega)} \leq C
$$

The compact embedding $W_{0}^{1, p}(\Omega) \hookrightarrow L^{p}(\Omega)$ and (28) ensure that there exists $u \in W_{0}^{1, p}(\Omega)$ such that, up to a subsequence,

$$
\begin{array}{ll}
u^{n} \rightarrow u & \text { strongly in } L^{p}(\Omega) \\
u^{n} \rightarrow u & \text { weakly in } W_{0}^{1, p}(\Omega) \\
u_{n} \rightarrow u & \text { a.e. in } \Omega .
\end{array}
$$

By Lemma 4, there exists $u^{\prime} \in W_{0}^{1, p}(\Omega), u^{\prime} \in[\underline{u}, \bar{u}]$ such that $u^{\prime}=T u$. We prove in what follows that $u$ is a fixed point of $T$ i.e. $u^{\prime}=u$.

From (27) and by the definition of $T$ we obtain

$$
\begin{array}{r}
(29) \int_{\Omega} a\left(x, \nabla u^{n+1}\right) \nabla\left(w-u^{n+1}\right) d x+J(w)-J\left(u^{n+1}\right) \geq \int_{\Omega} G\left(u^{n}\right)\left(w-u^{n+1}\right) \\
\text { for all } w \in W_{0}^{1, p}(\Omega) .
\end{array}
$$

Also, from $T u=u^{\prime}$, we have

$$
\int_{\Omega} a\left(x, \nabla u^{\prime}\right) \nabla\left(w-u^{\prime}\right) d x+J(w)-J\left(u^{\prime}\right) \geq \int_{\Omega} G(u) \cdot\left(w-u^{\prime}\right) d x
$$

Taking $w=u^{\prime}$ in (29) and $w=u^{n+1}$ in (30), we get

$$
\int_{\Omega} a\left(x, \nabla u^{n+1}\right) \nabla\left(u^{\prime}-u^{n+1}\right) d x+J\left(u^{\prime}\right)-J\left(u^{n+1}\right) \geq \int_{\Omega} G\left(u^{n}\right) \cdot\left(u^{\prime}-u^{n+1}\right) d x
$$


$\int_{\Omega} a\left(x, \nabla u^{\prime}\right) \nabla\left(u^{n+1}-u^{\prime}\right) d x+J\left(u^{n+1}\right)-J\left(u^{\prime}\right) \geq \int_{\Omega} G(u) \cdot\left(u^{n+1}-u^{\prime}\right) d x$.

So, by (29) and (30), $J\left(u^{\prime}\right)<\infty$ and $J\left(u^{n+1}\right)<\infty$. Summing up the last two inequalities we obtain

$$
\begin{aligned}
\int_{\Omega}\left(a\left(x, \nabla u^{\prime}\right)\right. & -a\left(x, \nabla u^{n+1}\right) \cdot \nabla\left(u^{\prime}-u^{n+1}\right) d x \\
& \leq \int_{\Omega}\left(G(u)-G\left(u^{n}\right)\right)\left(u^{\prime}-u^{n+1}\right) d x .
\end{aligned}
$$

Since $G$ is right continuous we have $G\left(u^{n}\right) \rightarrow G(u)$ in $\Omega$. We also have

$$
\left|G(u)-G\left(u^{n}\right)\right|\left(u-u^{n+1}\right) \leq 2(|G(\underline{u})|+|G(\bar{u})|)(|\underline{u}|+|\bar{u}|) \in L^{1}(\Omega) .
$$

By $\left(a_{2}\right)$ and the Lebesgue dominated convergence theorem, we deduce from (31) that

$$
\int_{\Omega}\left(a\left(x, \nabla u^{\prime}\right)-a\left(x, \nabla u^{n}\right) \cdot \nabla\left(u^{\prime}-u^{n}\right) d x \rightarrow 0 .\right.
$$

This implies that $\nabla u^{n} \rightarrow \nabla u^{\prime}$ a.e. in $\Omega$.

Relation (32) implies that (up to a subsequence)

$$
\left(a\left(x, \nabla u^{\prime}\right)-a\left(x, \nabla u^{n}\right)\right) \cdot \nabla\left(u^{\prime}-u^{n}\right) \rightarrow 0 \quad \text { a.e. } x \in \Omega .
$$

This leads to $\nabla u^{n} \rightarrow \nabla u^{\prime}$ a.e. in $\Omega$. Indeed, if not, there exists $x \in \Omega$ such that (up to a subsequence), $\nabla u^{n}(x) \rightarrow \xi \in \overline{\mathbf{R}}^{N}$ for $\xi \neq \nabla u^{\prime}$. Passing to the limit in (33) we obtain

$$
\left(a\left(x, \nabla u^{\prime}\right)-a(x, \xi)\right) \cdot\left(\nabla u^{\prime}-\xi\right)=0,
$$

which contradicts $\left(a_{2}\right)$. So, we have proved that $\nabla u^{n} \rightarrow \nabla u$. Using the fact that $u^{n} \rightarrow u$ weakly in $W_{0}^{1, p}(\Omega)$, we conclude that $\nabla u^{\prime}=\nabla u$, thus $u^{\prime}=u$. Replacing $u^{\prime}$ by $u$ in (30) we get

$$
\begin{aligned}
& \int_{\Omega} a(x, \nabla u) \cdot \nabla(w-u) d x+J(w)-J(u) \geq \int_{\Omega} G(u)(w-u) d x \\
& \text { for all } w \in W_{0}^{1, p}(\Omega) \text {. }
\end{aligned}
$$

Hence $u$ is a fixed point of $T$ and a solution for the problem $\left(P^{\prime}\right)$.

In order to prove that $u$ is a maximal solution of (3) with respect to the order interval $[\underline{u}, \bar{u}]$, take any other solution $\hat{u} \in[\underline{u}, \bar{u}]$ of the problem $\left(P^{\prime}\right)$. 
Then $\hat{u}$ is in particular a sub-solution satisfying $\hat{u} \leq \bar{u}$. Starting again the iteration (27) with $u^{0}=\bar{u}$ we obtain

$$
\hat{u} \leq \cdots \leq u^{n+1} \leq u^{n} \leq \cdot \leq u^{0}=\bar{u} .
$$

It follows that $\hat{u} \leq u$, which concludes our proof.

\section{REFERENCES}

[1] Barbu V., Precupanu Th., Convexity and Optimization in Banach Spaces, Sijthoff Noordoff, International Publishers, 1978.

[2] Brezis H., Integrales convexes dans les espaces de Sobolev, Israel J. Math., 13 (1972), 9-23.

[3] Brezis H., Opérateurs maximaux monotones et semi-groupes de contractions dans les espaces de Hilbert, North Holland, 1973.

[4] Carl S., A combined variational-monotone iterative method for elliptic boundary value problems with discontinuous nonlinearity, Appl. Anal., 43 (1992), 21-45.

[5] Lions J. L., Quelques méthodes de résolution des problèmes aux limites non linéaires, Gauthier-Villars, Paris, 1969.

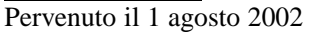

Simona Dăbuleanu - Vicențiu Rădulescu

Department of Mathematics University of Craiova 1100 Craiova, Romania E-mail:vicrad@yahoo.com 